\title{
A Quasilinear Model for Solute Transport under Unsaturated Flow
}

\author{
J.E. Houseworth and J. Leem ${ }^{\dagger}$
}

\section{Abstract}

This paper presents an analytical solution for solute transport under steady-state twodimensional unsaturated flow and transport conditions developed for the investigation of high-level radioactive waste disposal. The two-dimensional unsaturated flow problem is treated using the quasilinear flow method for a system with homogeneous material properties. Dispersion is modeled as isotropic and is proportional to the effective hydraulic conductivity. This leads to a quasilinear form for the transport problem in terms of a scalar potential that is analogous to the Kirchhoff potential for quasilinear flow. The solutions for both flow and transport scalar potentials take the form of Fourier series. The particular solution given here is for two sources of flow, with one source containing a dissolved solute. However, the solution method may easily be extended for any combination of flow and solute sources under steady-state conditions. The analytical results to multi-dimensional solute transport problems, which previously could only be solved numerically, also offer an additional way to benchmark numerical solutions.

\section{Introduction}

\footnotetext{
$\dagger$ J.E. Houseworth, Lawrence Berkeley National Laboratory, 1 Cyclotron Road Berkeley, CA 94720 (corresponding author: jehouseworth@lbl.gov)

J. Leem, Expat - Baku, Azerbaijan, P.O. Box 4381, Houston, TX 77210
} 
General analytical solutions for problems of transport during unsaturated flow in porous media are not possible because of the nonlinear relationships between water saturation, water pressure, and relative permeability. These non-linear relationships result in complex flow phenomena that cannot, in general, be evaluated by analytical methods for use in transport problems. Despite the severe restrictions for analytical methods, the special cases where analytical solutions are possible provide information concerning the behavior of the theoretical model without the uncertainties introduced by numerical approximations. For the same reason, analytical solutions are valuable for checking numerical solutions.

Various approximations that lead to linearized forms of the governing equations for unsaturated flow have been developed that are amenable to analytical solutions. However, only some of these cases have been extended to develop analytical results for transport problems. Most of the current available analytical methods for solute transport are restricted to steady or transient one-dimensional problems (e.g., Barry et al., 1991; Toride at al., 1993; Lessoff and Indelman, 2004; Vanderborght et al., 2005; Fityus et al., 1999). Two-dimensional solute transport under radial flow conditions was analyzed using analytical methods by Broadbridge et al. (2002).

The quasilinear form of the steady-state unsaturated flow problem has been used to investigate a variety of one-dimensional and multi-dimensional unsaturated flow problems through analytical methods (Pullan, 1990; Philip et al., 1989; Tartakovsky et al. 1999; Raats 1974, Raats et al. 1977a; Raats et al. 1977b). In particular, flow for a single 
strip source is presented in Warrick (2003). This form is developed using the Kirchhoff transformation and Gardner (1958) relationship between relative permeability and capillary pressure under steady-state flow conditions. Transient problems may also be investigated analytically assuming constant soil-water diffusivity. However, corresponding solutions for solute transport under multi-dimensional flow conditions are not available in the literature. Convective transport for unsaturated flow without dispersion is investigated by Raats (1974) and Raats et al. (1977a, b). Advectivedispersive transport solutions for homogeneous, unidirectional flow in one, two and three dimension are presented in Warrick (2003). The method developed here allows for the solution of two-dimensional, unsaturated flow and transport for steady-state flow and transport conditions. This method requires a particular form for the dispersion coefficient, homogeneous material properties, and simple domain boundaries. However, the method can accommodate any number of sources with different flow and solute concentration conditions. The solution method may be easily extended to three dimensions and, under more restrictive conditions, may be applied to steady-state flow with transient transport boundary conditions. The usefulness of the analytical solutions is also demonstrated by applying them to a specific example.

\section{Mathematical Formulation}

\section{Flow Problem}

The flow problem is developed for two sources are located along the upper boundary as shown in Figure 1. Source 1 enters the two-dimensional porous medium at a position $x_{1}$ 
with a flux, $q_{s 1}$. Source 2 enters at a position $x_{2}$ with a flux $q_{s 2}$. The remainder of the top boundary is a no-flow boundary. Both side boundaries are no-flow boundaries. The bottom boundary is a free-drainage boundary, which means that the water pressure gradients are zero along the bottom boundary.

The flow is governed by Darcy's law for unsaturated flow,

$\vec{q}=-K(\psi) \nabla \psi+K(\psi) \vec{\kappa}$

where $\vec{q}$ is the flux, $K(\psi)$ is the effective hydraulic conductivity to water, $\psi$ is the moisture potential, and $\vec{\kappa}$ is the unit vector in the vertical direction. Mass conservation for steady, incompressible flow is,

$\nabla \cdot \vec{q}=0$

Therefore,

$\nabla \cdot K \nabla \psi=\frac{\partial K}{\partial z}$

The boundary condition for the top boundary specifies the flux entering the domain from the two sources, 


$$
-K \frac{\partial \psi}{\partial z}(x, 0)+K(x, 0)=q_{s 1} H\left(x_{s 1}+x_{1}-x\right) H\left(x-x_{1}\right)+q_{s 2} H\left(x_{s 2}+x_{2}-x\right) H\left(x-x_{2}\right)
$$

where $H(x)$ is the step function $H(x)=1$ for $x>0, H(x)=0$ for $x<0$, and $H(0)=1 / 2$. The boundary condition on the bottom boundary is a "free gravity drainage" condition in which flow exits the bottom boundary by pure gravity-driven flow, i.e., the vertical water pressure gradient along the bottom boundary is zero,

$$
\frac{\partial \psi}{\partial z}\left(x, z_{m}\right)=0
$$

Because flow in the $\mathrm{x}$-direction requires a water pressure gradient, no flow conditions along each side boundary is obtained by setting the horizontal water pressure gradients along these boundaries to zero,

$$
\frac{\partial \psi}{\partial x}(0, z)=0
$$

$$
\frac{\partial \psi}{\partial x}\left(x_{m}, z\right)=0
$$

Introduce the Kirchhoff potential (Philip et al. 1989)

$$
\Phi(\psi)=\int_{-\infty}^{\psi} K(\vartheta) d \vartheta
$$


Using Equation (8) in Equation (3) gives,

$$
\nabla^{2} \Phi=\frac{\partial K}{\partial z}
$$

The constitutive relationship used for the effective permeability is the Gardner relationship (Gardner 1958),

$$
K(\psi)=K_{0} \exp \left\{\alpha\left(\psi-\psi_{0}\right)\right\}
$$

where $\alpha$ is the capillary strength of the material. Let

$$
\ell_{s}=\frac{2}{\alpha}
$$

be the sorptive length scale.

Using Equations (10) and (11) in Equation (8) gives,

$$
\Phi=\frac{\ell_{s}}{2} K
$$

Therefore, Equation (9) becomes, 


$$
\nabla^{2} \Phi=\frac{2}{\ell_{s}} \frac{\partial \Phi}{\partial z}
$$

Introduce the dimensional coordinates, $\xi=\frac{z}{\ell_{s}}, \eta=\frac{x}{\ell_{s}}$, and $\Phi_{d}=\frac{\Phi}{q_{s 1} \ell_{s}} \quad$ to give,

$$
\nabla_{d}^{2} \Phi_{d}=2 \frac{\partial \Phi_{d}}{\partial \xi}
$$

where $\nabla_{d} \equiv \ell_{s} \nabla$. Introduce the change of variables,

$$
\Phi_{d}=\chi \exp (\xi)
$$

to give,

$$
\nabla_{d}^{2} \chi=\chi
$$

Using Equations (1), (8), (12), and (15), the dimensionless flux vector may be expressed in terms of the dimensionless Kirchhoff potential or the transformed potential function, $\chi \exp (\xi)$, to be,

$\vec{q}_{d}=2 \Phi_{d} \vec{\kappa}-\nabla_{d} \Phi_{d}=\exp (\xi)\left(\chi \vec{\kappa}-\nabla_{d} \chi\right)$

where $\vec{q}_{d}=\frac{\vec{q}}{q_{s 1}}$. 
Let the dimensionless boundary coordinates be $\omega=\frac{z_{m}}{\ell_{s}}, \sigma=\frac{x_{m}}{\ell_{s}}$. The boundary conditions become,

$$
\chi(\eta, 0)-\frac{\partial \chi}{\partial \xi}(\eta, 0)=H\left(\eta_{s 1}+\eta_{1}-\eta\right) H\left(\eta-\eta_{1}\right)+q_{s 2 d} H\left(\eta_{s 2}+\eta_{2}-\eta\right) H\left(\eta-\eta_{2}\right)
$$

$$
\frac{\partial \chi}{\partial \xi}(\eta, \omega)+\chi(\eta, \omega)=0
$$

$$
\frac{\partial \chi}{\partial \eta}(0, \xi)=0
$$

$$
\frac{\partial \chi}{\partial \eta}(\sigma, \xi)=0
$$

\section{Transport Problem}

The transport problem is developed for the same two sources located along the upper boundary as shown in Figure 1. However, only Source 1 carries solute. The steady-state flow from the source has a Darcy velocity, $q_{s 1}$ and a solute concentration of $C_{s 1}$. The steady-state flow from the other source has a Darcy velocity, $q_{s 2}$, and radionuclide concentration, $C_{s 2}=0$. The remainder of the top boundary is a no-flux boundary. Both side boundaries are no-flux boundaries. The bottom boundary is a no diffusive flux boundary (advection only), which means that the solute concentration gradients in the z- 
direction are zero along the bottom boundary. Given these boundary conditions, transport in the two-dimensional porous medium is governed by a balance of advective and dispersive fluxes (mass conservation),

$\vec{q} \cdot \nabla C=\nabla \cdot(\theta D \nabla C)$

where $\theta$ is the water content and $D$ is the dispersion coefficient.

The top boundary conditions specify the radionuclide mass flux entering the domain,

$q(x, 0) C(x, 0)-\theta(x, 0) D(x, 0) \frac{\partial C(x, 0)}{\partial z}=\dot{M}_{s 1} H\left(x_{s 1}+x_{1}-x\right) H\left(x-x_{1}\right)$

where $H(x)$ is the step function $H(x)=1$ for $x>0, H(x)=0$ for $x<0$, and $H(0)=1 / 2$. The radionuclide mass flux from Source 1 is $\dot{M}_{s 1}=q_{s 1} C_{s 1}$. The bottom boundary condition specifies that solute mass exits the domain through advective transport only, i.e., the dispersive flux in the z-direction along the bottom boundary is zero. Therefore, the vertical concentration gradient along the bottom boundary is set to zero.

$$
\frac{\partial C}{\partial z}\left(x, z_{m}\right)=0
$$

Radionuclide mass flux in the $\mathrm{x}$-direction along the side boundaries is specified to be zero. This is accomplished by setting the concentration gradients in the $\mathrm{x}$-direction along 
these boundaries to zero, i.e., zero dispersive flux in the $\mathrm{x}$-direction along these boundaries. Note that the advective flux is also zero because the flow analysis specifies zero flow across these boundaries (see boundary conditions in Equations (6) and (7)).

$$
\begin{aligned}
& \frac{\partial C}{\partial x}(0, z)=0 \\
& \frac{\partial C}{\partial x}\left(x_{m}, z\right)=0
\end{aligned}
$$

The Darcy water flux, $\vec{q}$, is defined by the flow solution. To proceed, the dispersion coefficient needs to be specified. In its most general form, the dispersion coefficient is an anisotropic tensor (Bear 1972). For unsaturated flow, the dispersivities are also found to be functions of water saturation (Toride at al. 2003). Only a limited amount of experimental data is available for dispersion in unsaturated flow, and little is known about dispersion for two or three-dimensional flow conditions. Given these uncertainties, the dispersion tensor will be simplified to be a scalar function of water saturation. The following simplification allows for analytical treatment of transport,

$\theta D=\Phi(\psi)$

where $\Phi$ is the Kirchhoff potential. This form for the dispersion coefficient is shown to be consistent with experimental data later in this paper. It should be pointed out, however, that the representation of dispersion given in Equations (27) is not expected to 
be accurate for conditions where molecular diffusion is important relative to hydrodynamic dispersion.

Using Equation (27), Equation (22) becomes,

$\vec{q} \cdot \nabla C=\nabla \cdot(\Phi \nabla C)$

Putting Equation (28) into nondimensional form gives,

$\vec{q}_{d} \cdot \nabla_{d} C_{d}=\nabla_{d} \cdot\left(\Phi_{d} \nabla_{d} C_{d}\right)$

where $C_{d}=\frac{q_{s 1} C}{\dot{M}_{s 1}}$.

Equation (29) may also be expressed as,

$\nabla_{d} \cdot\left(\vec{q}_{d} C_{d}\right)=\nabla_{d} \cdot\left(\Phi_{d} \nabla_{d} C_{d}\right)$

because of the mass conservation for steady, incompressible flow, $\nabla_{d} \bullet \vec{q}_{d}=0$.

Substituting for $\vec{q}_{d}$ from Equation (17) into Equation (30) gives, 


$$
\nabla_{d}^{2}\left(\Phi_{d} C_{d}\right)=2 \frac{\partial\left(\Phi_{d} C_{d}\right)}{\partial \xi}
$$

Therefore, the transport problem is now expressed in terms of the dimensionless scalar potential,

$$
\Omega \equiv \Phi_{d} C_{d}
$$

Equation (31) becomes,

$$
\nabla_{d}^{2} \Omega=2 \frac{\partial \Omega}{\partial \xi}
$$

Using the transformation

$$
\Omega=\Theta \exp (\xi)
$$

Equation (34) transforms to the modified Helmholtz equation,

$$
\nabla_{d}^{2} \Theta=\Theta
$$

and boundary conditions (23) through (26) become, 


$$
\Theta(\eta, 0)-\frac{\partial \Theta}{\partial \xi}(\eta, 0)=H\left(\eta_{s 1}+\eta_{1}-\eta\right) H\left(\eta-\eta_{1}\right)
$$

$$
\frac{\partial \Theta}{\partial \xi}(\eta, \omega)+\Theta(\eta, \omega)=0
$$

$$
\frac{\partial \Theta}{\partial \eta}(0, \xi)=0
$$

$$
\frac{\partial \Theta}{\partial \eta}(\sigma, \xi)=0
$$

\section{Mathematical Solutions}

\section{Flow Problem}

Equations (16) with boundary conditions (18) through (21) may be solved by the method of separation of variables (e.g., Weinberger 1965). The solution is given by,

$$
\begin{aligned}
\chi(\eta, \xi)=\sum_{\ell=0}^{\infty} & \frac{2\left[\sin \left[\lambda_{\ell}\left(\eta_{s 1}+\eta_{1}\right)\right]-\sin \left(\lambda_{\ell} \eta_{1}\right)+q_{s 2 d}\left\{\sin \left[\lambda_{\ell}\left(\eta_{s 2}+\eta_{2}\right)\right]-\sin \left(\lambda_{\ell} \eta_{2}\right)\right\}\right]}{(1+2 H(-\ell)) \lambda_{\ell} \sigma} \cos \left(\lambda_{\ell} \eta\right) \\
& \cdot \frac{\Lambda_{\ell} \cosh \left\{\Lambda_{\ell}(\omega-\xi)\right\}+\sinh \left\{\Lambda_{\ell}(\omega-\xi)\right\}}{2 \Lambda_{\ell} \cosh \left(\Lambda_{\ell} \omega\right)+\left(\Lambda_{\ell}^{2}+1\right) \sinh \left(\Lambda_{\ell} \omega\right)}
\end{aligned}
$$

where $\lambda_{\ell}=\frac{\ell \pi}{\sigma}$ and $\Lambda_{\ell}=\sqrt{1+\lambda_{\ell}^{2}}$. The flux field is computed using equation (17) to give, 


$$
\begin{gathered}
q_{\xi d}=\exp (\xi) \sum_{\ell=0}^{\infty} \frac{2\left[\left\{\sin \left[\lambda_{\ell}\left(\eta_{s 1}+\eta_{1}\right)\right]-\sin \left(\lambda_{\ell} \eta_{1}\right)\right\}+q_{s 2 d}\left\{\sin \left[\lambda_{\ell}\left(\eta_{s 2}+\eta_{2}\right)\right]-\sin \left(\lambda_{\ell} \eta_{2}\right)\right\}\right]}{(1+2 H(-\ell)) \lambda_{\ell} \sigma} \cos \left(\lambda_{\ell} \eta\right) \\
\bullet \exp \left(-\Lambda_{\ell} \xi\right) \frac{\left(\Lambda_{\ell}+1\right)^{2}-\left(\Lambda_{\ell}-1\right)^{2} \exp \left\{-2 \Lambda_{\ell}(\omega-\xi)\right\}}{\left(\Lambda_{\ell}+1\right)^{2}-\left(\Lambda_{\ell}-1\right)^{2} \exp \left\{-2 \Lambda_{\ell} \omega\right\}}
\end{gathered}
$$

$$
\begin{gathered}
q_{\eta d}=\exp (\xi) \sum_{\ell=0}^{\infty} \frac{2\left[\left\{\sin \left[\lambda_{\ell}\left(\eta_{s 1}+\eta_{1}\right)\right]-\sin \left(\lambda_{\ell} \eta_{1}\right)\right\}+q_{s 2 d}\left\{\sin \left[\lambda_{\ell}\left(\eta_{s 2}+\eta_{2}\right)\right]-\sin \left(\lambda_{\ell} \eta_{2}\right)\right\}\right]}{(1+2 H(-\ell)) \sigma} \sin \left(\lambda_{\ell} \eta\right) \\
\cdot \exp \left(-\Lambda_{\ell} \xi\right) \frac{\left(\Lambda_{\ell}+1\right)+\left(\Lambda_{\ell}-1\right) \exp \left\{-2 \Lambda_{\ell}(\omega-\xi)\right\}}{\left(\Lambda_{\ell}+1\right)^{2}-\left(\Lambda_{\ell}-1\right)^{2} \exp \left\{-2 \Lambda_{\ell} \omega\right\}}
\end{gathered}
$$

\section{Transport Problem}

The problem given in Equations (36) through (40) is the same as for the dimensionless flow potential with $q_{\mathrm{s} 2}=0$ (Equation (16) and boundary conditions (18) through (21)). Therefore, the solution for the scaled dimensionless transport potential may be obtained directly from Equation (41) to give,

$$
\begin{aligned}
\Theta(\eta, \xi)=\sum_{\ell=0}^{\infty} \frac{2\left[\sin \left\{\lambda_{\ell}\left(\eta_{s 1}+\eta_{1}\right)\right\}-\sin \left(\lambda_{\ell} \eta_{1}\right)\right]}{(1+2 H(-\ell)) \lambda_{\ell} \sigma} \cos \left(\lambda_{\ell} \eta\right) \\
\\
\bullet \exp \left(-\Lambda_{\ell} \xi\right) \frac{\left(\Lambda_{\ell}+1\right)+\left(\Lambda_{\ell}-1\right) \exp \left\{-2 \Lambda_{\ell}(\omega-\xi)\right\}}{\left(\Lambda_{\ell}+1\right)^{2}-\left(\Lambda_{\ell}-1\right)^{2} \exp \left(-2 \Lambda_{\ell} \omega\right)}
\end{aligned}
$$

Furthermore, the solute concentration is given by, 


$$
C_{d}(\eta, \xi)=\frac{\Theta(\eta, \xi)}{\chi(\eta, \xi)}
$$

\section{Relationship between the Kirchhoff Potential and Dispersion Coefficient}

The transformation of the transport equation into a form expressed in terms of a scalar potential (Equations (35) and (37)) requires that the dispersion coefficient times the water content equals the Kirchhoff potential, as given in Equation (27). Longitudinal dispersion in unsaturated flow systems has been measured under conditions of uniform, onedimensional, unsaturated flow (Toride et al. 2003). Hydraulic parameters for the granular media were based on the measured moisture potential as a function of water content fit with the van Genuchten (1980) parameterization. Table 1 gives the hydraulic parameters were determined for the test system:

Table 1.Hydrologic Parameters from Toride et al (2003)

\begin{tabular}{|l|r|}
\hline parameter & value \\
\hline maximum water content & 0.35 \\
\hline residual water content & 0.05 \\
\hline van Genuchten $m$ & 0.808 \\
\hline van Genuchten $\alpha_{v G}$ & $5 \mathrm{~m}^{-1}$ \\
\hline saturated hydraulic conductivity & $6.36 \times 10^{-5} \mathrm{~m} / \mathrm{s}$ \\
\hline
\end{tabular}


The mathematical model used for hydraulic conductivity as a function of moisture potential is the Gardner relationship, as given in Equation (10). Therefore, the data provided by Toride et al. (2003) were used to parameterize the Gardner relationship. Hydraulic conductivity was computed as a function of moisture potential using the van Genuchten model and the parameters in Table 1. The values are computed at the saturation levels measured in the unsaturated flow and transport experiments conducted by Toride et al. (2003). These values are shown as points on the log-linear plot given in Figure 2. The Gardner model plots as a straight line on a log-linear scale, therefore, the slope of the straight-line fit to the points gives the value of the Gardner capillary strength parameter, $\alpha$. The value of the Gardner capillary strength parameter is found to be $37.4 \mathrm{~m}^{-1}$, which may also be expressed as the sorptive length scale, $\ell_{s}=2 / \alpha=0.0535 \mathrm{~m}$.

The Kirchhoff potential is given by Equation (12), which is dimensionally equivalent to a diffusion or dispersion coefficient. A comparison of the magnitude of the Kirchhoff potential computed from Equation (12), versus the experimentally determined longitudinal dispersion multiplied times the water content from Toride et al. (2003) is given in Figure 3. This figure shows that the Kirchhoff potential provides a reasonable estimate for the longitudinal dispersion as a function of hydrologic conditions. Because transverse dispersion is generally smaller than longitudinal dispersion under saturated flow conditions (Bear, 1972), the same may be expected for unsaturated systems. If so, the use of the Kirchhoff potential as an isotropic dispersion coefficient likely overestimates lateral dispersion. Also, note that data for dispersion under more complex unsaturated flow patterns are not available and remain uncertain. 


\section{Specific Example for Flow and Transport in a Granular Invert}

The example presented here was developed for investigations of geologic disposal of high-level radioactive waste. The radioactive waste is contained in a waste package that is a cylindrical vessel approximately $2 \mathrm{~m}$ in diameter and $5 \mathrm{~m}$ long. The waste package is located in a roughly horizontal, underground drift having a circular cross section. The base of the drift is filled with granular crushed rock called the invert. The waste package is placed in the drift with its cylindrical axis parallel to the axis of the drift. If a waste package is breached, water may enter the waste package and contact, dissolve, and transport the waste. The water flowing through the waste package enters the invert and mixes with water flowing adjacent to the waste package. The mathematical model presented in this paper is for a two-dimensional cross-section oriented orthogonal to the axes of the waste package and drift to investigate flow and transport behavior in the invert. The rectangular geometry for the invert shown in Figure 1 is an approximation used to simplify the model boundary conditions.

The parameters required for flow and transport calculations are, the depth and width of the domain, $z_{m}$ and $x_{m}$, respectively, the locations of the two source regions, $x_{1}$ and $x_{2}$, the sizes of the two source regions, $x_{s 1}$ and $x_{s 2}$, the water fluxes for the two source regions, $q_{s 1}$ and $q_{s 2}$, the capillary strength parameter, $\alpha$. The solute mass flux for the source region, $\dot{M}_{s 1}$, is also needed if mass concentrations are required from the transport 
calculation. However, in this analysis only relative concentrations are computed using Equation (44), so $\dot{M}_{s 1}$ is not needed.

For the specific case presented here, Source 1 is on the left-hand boundary and Source 2 occupies a more substantial region, $0.59 \mathrm{~m}$, to the right of Source 1 . This is a symmetry model where the left-hand boundary represents the center of the waste emplacement drift. Source 1, represents flow through the waste package, while water from Source 2, located to the right of Source 1 represents water that has not contacted the waste. Parameters used for the calculation are given in Table 2 .

Table 2. Parameters for Invert Flow and Transport Problem

\begin{tabular}{|l|l|}
\hline Parameter Symbol & Parameter Value \\
\hline$x_{1}$ & $0 \mathrm{~m}$ \\
\hline$x_{2}$ & $1 \mathrm{~m}$ \\
\hline$x_{s 1}$ & $0.01 \mathrm{~m}$ \\
\hline$x_{s 2}$ & $0.59 \mathrm{~m}$ \\
\hline$x_{m}$ & $1.59 \mathrm{~m}$ \\
\hline$z_{m}$ & $0.864 \mathrm{~m}$ \\
\hline$q_{s 1}$ & $9,800 \mathrm{~mm} / \mathrm{yr}$ \\
\hline$q_{s 2}$ & $66 \mathrm{~mm} / \mathrm{yr}$ \\
\hline$\alpha$ & $7.22 \mathrm{~m}^{-1}$ \\
\hline
\end{tabular}

\section{Results for Invert Flow and Transport Problem}


The solute source flow into the domain, Source 1, is a narrow 2-cm zone at the left hand boundary. The solute source water mixes with a second water source, Source 2, which is distributed uniformly over a larger portion of the domain along the right-hand boundary.

The streamlines are shown in Figure (4). A uniform flux of $66 \mathrm{~mm} /$ year is introduced through Source 2 along the top of the domain from a horizontal coordinate of $1 \mathrm{~m}$ to 1.59 m. The stronger Source 1 flow is seen in Figure (4) to intrude beneath the weaker and more diffuse Source 2.

The results of this flow pattern on the relative concentrations are shown in Figure (5). The separation streamline between the two sources is indicated on the figure, which would be the limiting boundary for transport of solutes issued from Source 1 under a nondispersive transport process. A broad mixing zone is found, where the maximum extent of the $1 \%$ mixing zone spans the horizontal dimension of the domain.

Convergence of the series depends on the parameter values as well as the values of the flux and concentration. Regions where flux is small may require thousands of terms to achieve $1 \%$ accuracy whereas regions with larger values can achieve even greater accuracy with less than 100 terms. The infinite series were truncated at 10,000 terms for the calculations presented here. Problems with numerical accuracy become significant for calculations of relative concentrations if $\alpha$ is on the order of $100 \mathrm{~m}^{-1}$ or larger. Large values of $\alpha$ lead to relatively simple, near-vertical flow patterns with low solute dispersion. The numerical problem for large values of $\alpha$ occurs because of the nature of 
the concentration solution given in Equation (45). For large values of $\alpha$, large areas of the invert have very small values of both the transport potential, $\Theta$, and flow potential, $\chi$. These small potentials are generated from the summation of terms of much greater magnitude that nearly cancel. This situation leads to a loss in numerical accuracy. For the flow fields, the lack of accuracy is not critical because as $\chi$ becomes small, so do the fluxes. Therefore areas where $\chi$ loses accuracy also have nearly zero flux. But for concentration this is not the case. As both $\Theta$ and $\chi$ become small, the relative concentration, which is computed as the ratio of these potentials, can be any value ranging between 0 and 1 . The loss in accuracy can affect significant ranges of the relative concentration, not just values near zero.

\section{Possible Extension to Three Dimensions and Transient Transport}

Three-dimensional steady-state flow and transport problems are easily treated using the methods discussed above without any further restrictions than needed for the twodimensional problem. The solution results in a double summation representing the Fourier modes for the two transverse directions.

Unsteady transport in a steady-state flow field may be treated using an extension of the same methodology. In this case, however, an additional restriction is required. The unsteady term contains the product of the water saturation and the solute concentration. The effective hydraulic conductivity must be proportional to the water saturation to relate this term into the scalar potential for transport given in Equation (32). This is analogous to the approximation of a constant hydraulic diffusivity used for analytical treatment of 
unsteady quasilinear flow problems. With this approximation, a quasilinear, unsteady transport equation may be derived in terms of the scalar potential for transport. A similar restriction is required to treat simple decay processes under steady or unsteady transport conditions.

\section{Conclusions}

An analytical method has been developed for transport under quasilinear flow for a particular parameterization of the dispersion coefficient. This parameterization has been shown to be plausible, if not quantitatively exact for a particular problem. Although the domain geometry required for analytical treatment is restrictive, virtually any configuration of water and solute source terms may be treated under two-dimensional or three-dimensional steady flow conditions. The main uses of the solution may be for testing numerical models and for poorly characterized problems where simplified parameterization may be appropriate.

\section{Acknowledgements}

The authors would like to thank H. H. Liu for his review of this paper. This manuscript has been authored by Lawrence Berkeley National Laboratory under Contract No. DEAC02-05CH11231 with the U.S. Department of Energy. The United States Government retains and the publisher, by accepting the article for publication, acknowledges that the United States Government retains a non-exclusive, paid-up, irrevocable, world-wide 
license to publish or reproduce the published form of this manuscript, or allow others to do so, for United States Government purposes.

The views expressed in this article are those of the author[s] and do not necessarily reflect the views or policies of the United States Department of Energy or The Regents of the University of California. 


\section{References}

Barry, D.A., G.C. Sander, and I.R. Phillips, 1991. Modelling solute transport, chemical adsorption and cation exchange, pp. 913-918, Proc. Int. Hydrol. Water Resour. Symposium, Institution of Engineers, Perth, Australia 92-4 October, 1991.

Bear, J. 1972. Dynamics of Fluids in Porous Media. Environmental Science Series. Biswas, A.K., ed. New York, New York: Elsevier.

Broadbridge, P.; J.R. Moitsheki; M. P. Edwards. 2002. Analytical Solutions for TwoDimensional Solute Transport with Velocity-Dependent Dispersion. in Environmental Mechanics Water, Mass and Energy Transfer in the Biosphere,Geophysical Monograph Series, The Philip Volume. Ed. P.A.C. Raats, D. Smiles, and A.W. Warrick. Geophysical Monograph Series, Vol. 129. pp. 145-153.

Fityus, S.G.; Smith, D.W.; Booker, J.R. 1999. Contaminant transport through an unsaturated soil liner beneath a landfill. Canadian Geotechnical Journal. Vol. 36. pp. $330-354$

Gardner, W. R. 1958. Some Steady-State Solutions of the Unsaturated Moisture Flow Equation with Application to Evaporation from a Water Table., Soil Science, 85, 228232.

Lessoff, S.C. and P. Indelman. 2004. Analytical model of solute transport by unsteady unsaturated gravitational infiltration. J Contam Hydrol. Aug; 72(1-4):85-107. 
Philip, J.R.; Knight, J.H.; and Waechter, R.T. 1989. Unsaturated Seepage and Subterranean Holes: Conspectus, and Exclusion Problem for Circular Cylindrical Cavities. Water Resources Research, 25, (1), 16-28. Washington, D.C.: American Geophysical Union.

Pullan, A. J. 1990. The Quasilinear Approximation for Unsaturated Porous Media Flow. Water Resources Research, Vol. 26, No. 6, pp. 1219-1234.

Raats, P.A.C. 1974. Steady Flows of Water and Salt in Uniform Soil Profiles with Plant Roots. Soil Science Society of America Journal, Vol. 38. pp. 717-722.

Raats, P.A.C. 1977a. Convective transport of solutes by steady flows I. General theory. Agricultural Water Management, Vol. 1, No. 3, pp. 201-218.

Raats, P.A.C. 1977b. Convective transport of solutes by steady flows I I. Specific flow problems. Agricultural Water Management, Vol. 1, No. 3, pp. 219-232.

Tartakovsky, D.M., Neuman, S.P., Lu, Z. 1999, Conditional stochastic averaging of steady state unsaturated flow by means of Kirchhoff transformations. Water Resources Research, v. 35, n. 3, p. 731-745.

Toride, N., F.J. Leij, and M.Th. van Genuchten. 1993. A comprehensive set of analytical solutions for nonequilibrium solute transport with first-order decay and zero-order production, Water Resour. Res., 29, 2167-2182.

Toride, Nobuo; Inoue, Mitsuhiro; and Leij, Feike. 2003. "Hydrodynamic Dispersion in an Unsaturated Dune Sand", Soil Sci. Soc. Am. J. 67:703-712. 
Vanderborght, J.; R. Kasteel; M. Herbst; M. Javaux; D. Thiéry; M. Vanclooster; C. Mouvet; and H. Vereecken. 2005. A Set of Analytical Benchmarks to Test Numerical Models of Flow and Transport in Soils. Vadose Zone Journal 4:206-221.

van Genuchten, M.T. 1980. "A Closed-Form Equation for Predicting the Hydraulic Conductivity of Unsaturated Soils." Soil Science Society of America Journal, 44, (5), 892-898. Madison, Wisconsin: Soil Science Society of America.

Warrick, A.W. 2003. Soil Water Dynamics. Oxford University Press.

Weinberger, H. F. 1965. A First Course in Partial Differential Equations with Complex Variables and Transform Methods. Blaisdell Publishing Company, New York. 


\section{Nomenclature}

$C \equiv$ solute mass concentration

$C_{d}=\frac{q_{s 1} C}{\dot{M}_{s 1}} \equiv$ dimensionless concentration

$C_{s 1} \equiv$ solute mass concentration in source 1

$D \equiv$ dispersion coefficient

$H(x) \equiv$ step function, $H(x)=1$ for $x>0, H(x)=0$ for $x<0$, and $H(0)=1 / 2$

$K\left(S_{w}\right) \equiv$ effective hydraulic conductivity

$\ell_{s}=\frac{2}{\alpha} \equiv$ sorptive length scale

$\dot{M}_{s 1}=q_{s 1} C_{s 1} \equiv$ solute mass flux from source 1

$\vec{q} \equiv$ darcy water flux

$\vec{q}_{d}=\frac{\vec{q}}{q_{s 1}} \equiv$ dimensionless flux

$q_{\eta d} \equiv$ dimensionless flux in horizontal direction

$q_{\xi d} \equiv$ dimensionless flux in vertical direction

$q_{s 1} \equiv$ water flux for source 1

$q_{\mathrm{s} 2} \equiv$ water flux for source 2

$q_{s 2 d}=\frac{q_{s 2}}{q_{s 1}} \equiv$ dimensionless water flux for source 2

$S_{w} \equiv$ water saturation

$x \equiv$ horizontal coordinate 
$x_{1} \equiv$ position of source 1

$x_{s 1} \equiv$ size of source 1

$x_{2} \equiv$ position of source 2

$x_{s 2} \equiv$ size of source 2

$Z \equiv$ vertical coordinate

$x_{m} \equiv$ horizontal boundary coordinate

$z_{m} \equiv$ vertical boundary coordinate

$\alpha \equiv$ capillary strength

$\lambda_{\ell}=\frac{\ell \pi}{\sigma}$

$\Lambda_{\ell}=\sqrt{1+\lambda_{\ell}^{2}}$

$\xi=\frac{Z}{\ell_{s}} \equiv$ dimensionless vertical coordinate

$\vec{\kappa} \equiv$ unit vector in vertical direction

$\eta=\frac{x}{\ell_{s}} \equiv$ dimensionless horizontal coordinate

$\eta_{1} \equiv$ dimensionless position of source 1

$\eta_{s 1} \equiv$ dimensionless size of source 1

$\eta_{2} \equiv$ dimensionless position of source 2

$\eta_{s 2} \equiv$ dimensionless size of source 2

$\Phi(\psi) \equiv$ Kirchhoff potential

$\Phi_{d}=\frac{\Phi}{q_{s 1} \ell_{s}} \equiv$ dimensionless Kirchhoff potential 


$$
\begin{aligned}
& \theta \equiv \text { water content } \\
& \Theta=\Omega \exp (-\xi) \equiv \text { scaled dimensionless transport potential } \\
& \psi\left(S_{w}\right) \equiv \text { moisture potential } \\
& \sigma=\frac{x_{m}}{\ell_{s}} \equiv \text { dimensionless horizontal boundary coordinate } \\
& \chi=\Phi_{d} \exp (-\xi) \equiv \text { scaled dimensionless flow potential } \\
& \omega=\frac{z_{m}}{\ell_{s}} \equiv \text { dimensionless vertical boundary coordinate } \\
& \Omega \equiv \Phi_{d} C_{d} \equiv \text { dimensionless transport potential }
\end{aligned}
$$

Figure 1. Domain and boundary conditions for flow

Figure 2. Effective Permeability - Moisture Potential Plot for Toride et al. (2003) Experiments.

Figure 3. Comparison of Kirchhoff Potential with Dispersion as a Function of Water Content using Toride et al. (2003) Experimental Results.

Figure 4. Streamlines for Invert Transport Problem

Figure 5. Relative Concentration Contours for Invert Transport Problem 


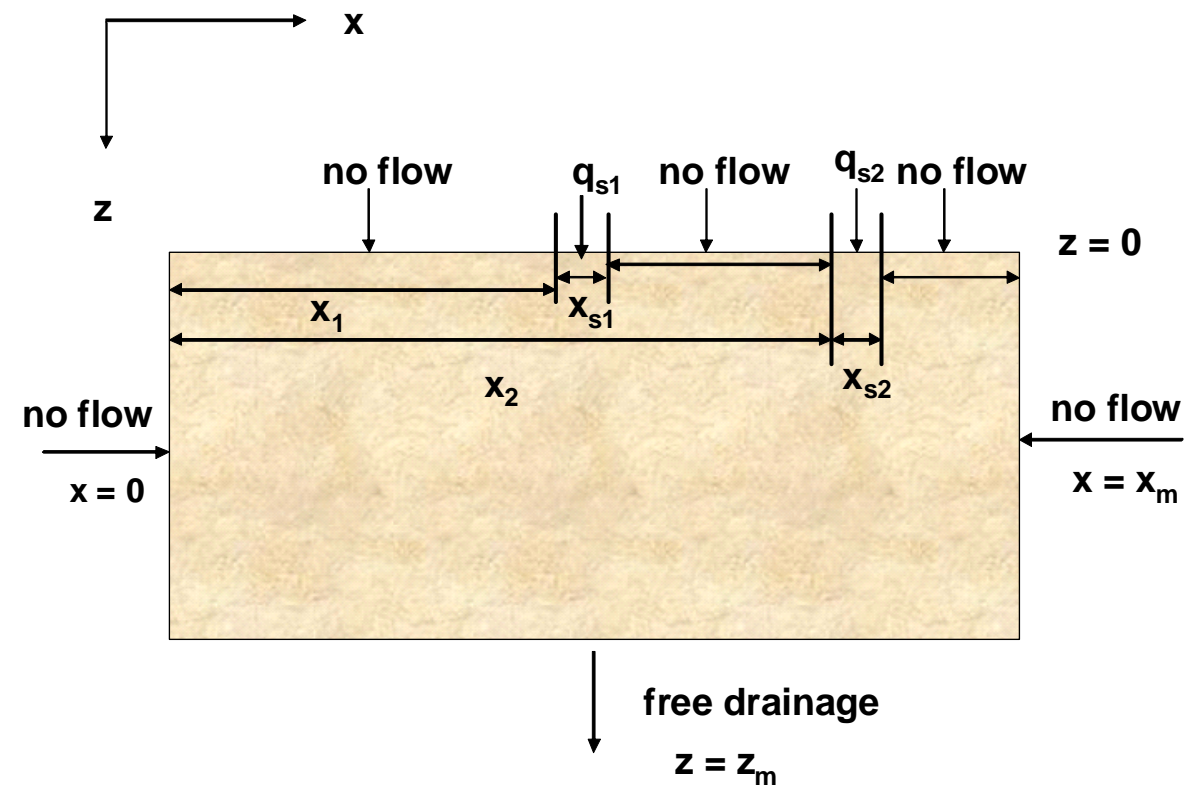

Figure 1 


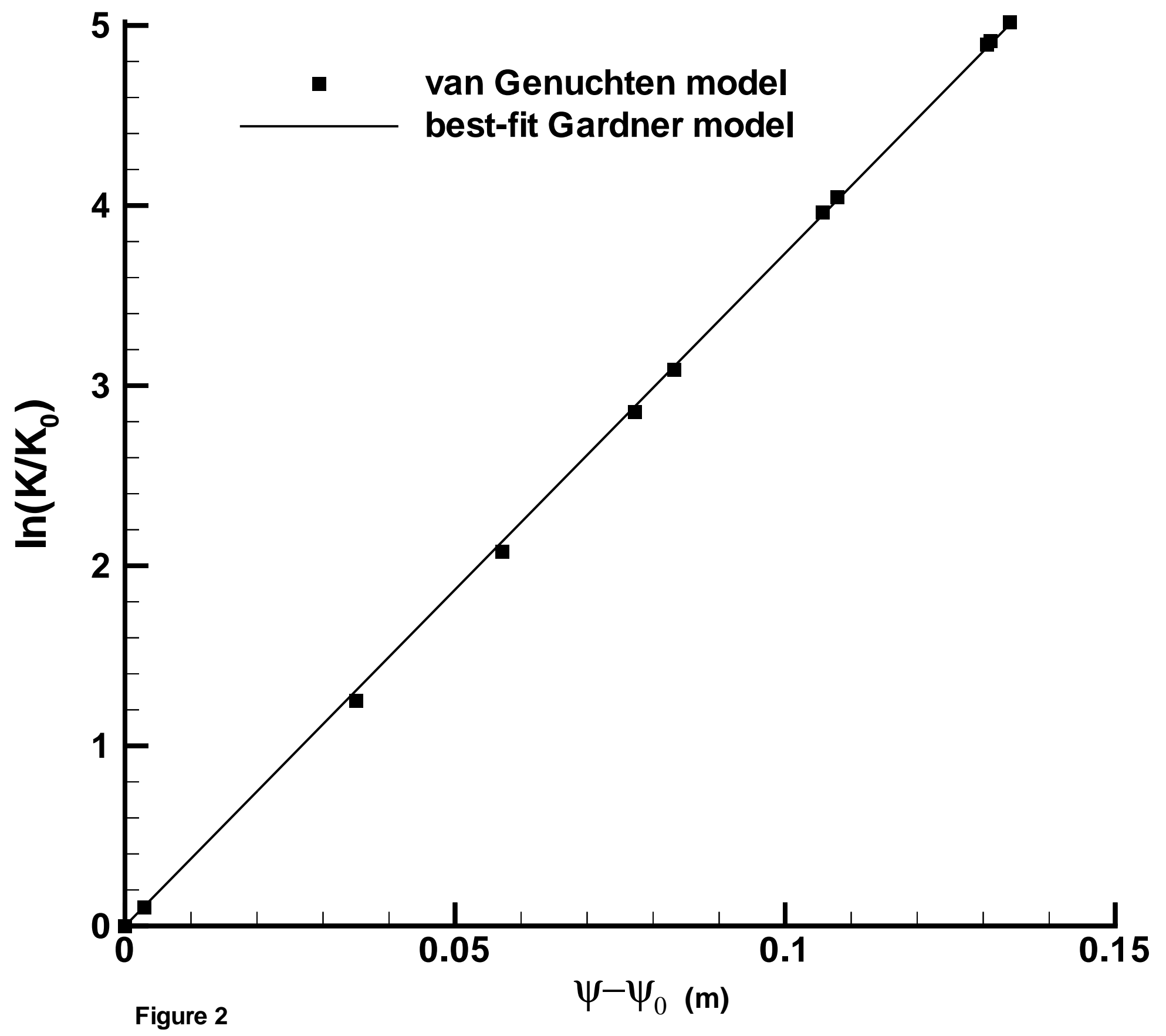




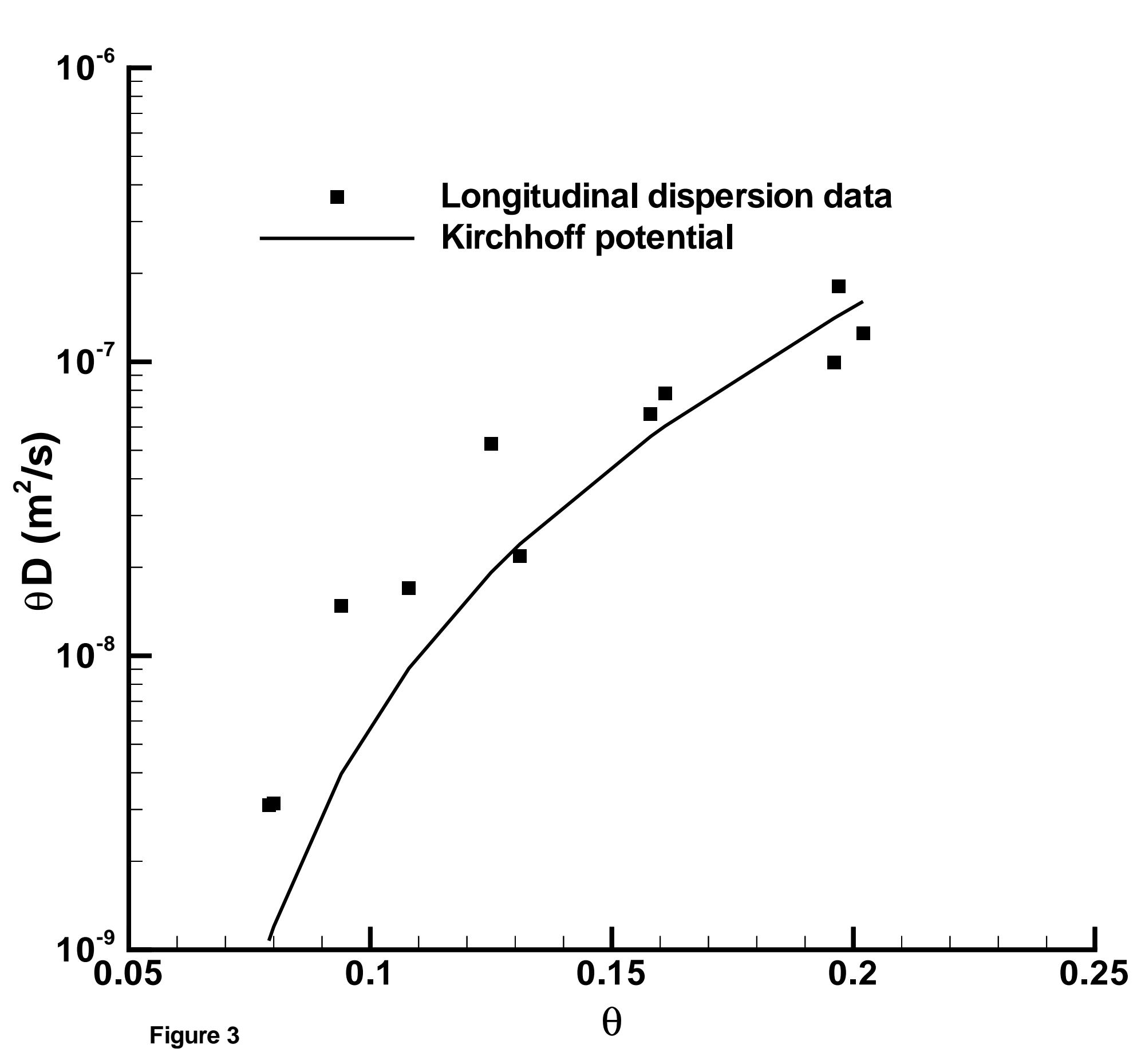

Figure 3

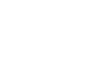




\section{Horizontal Coordinate (m)}

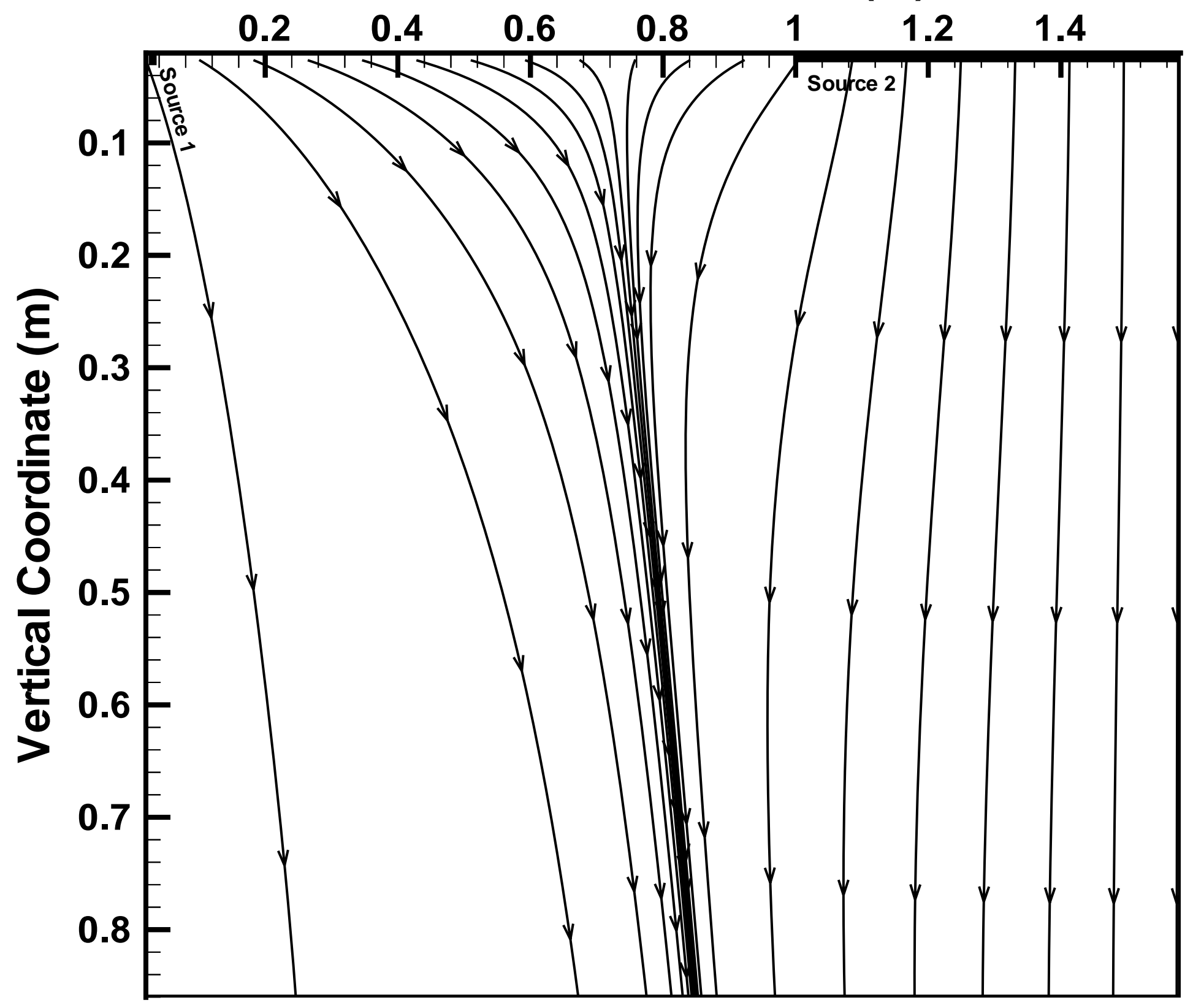

Figure 4 


\section{Horizontal Coordinate (m)}

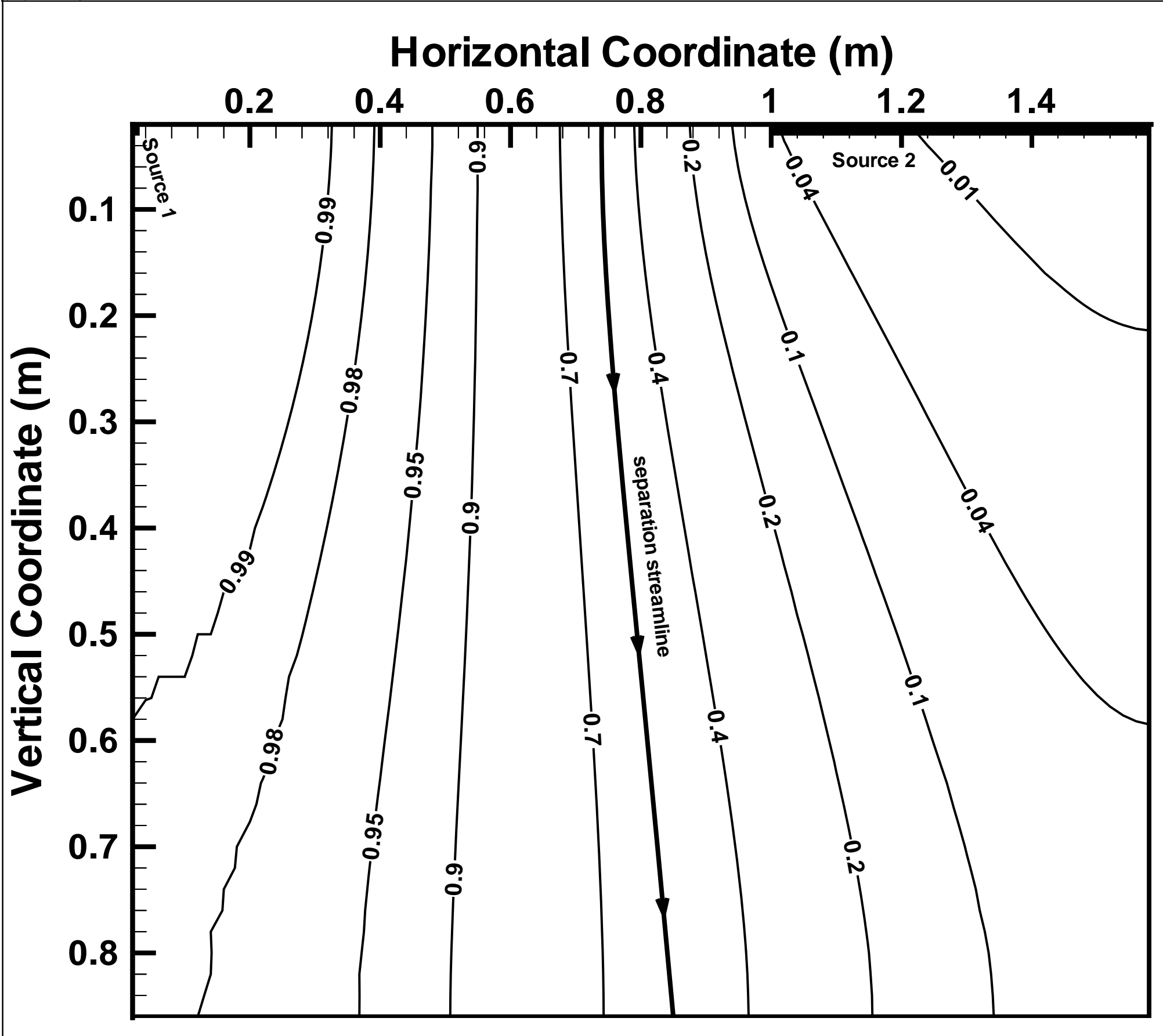

Figure 5

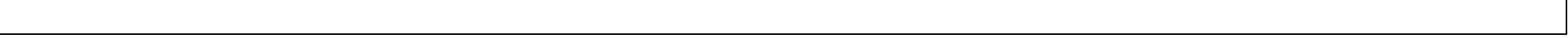

